\title{
Effects of drying methods on total phenolic contents and antioxidant capacity of the pomelo (Citrus grandis (L.) Osbeck) peels
}

\begin{abstract}
Pomelo (Citrus grandis (L.) Osbeck) is an important cultivar of the Citrus genus, which contains a number of nutrients advantageous to human health. Non-edible parts of pomelo consist of flavedo, albedo, lamella and waste obtained from juice extraction (pulp waste). The peels are also thought to be part of waste products, which generally being discarded. Therefore, the objective of the present study was to evaluate changes in total phenolic contents (TPC) and antioxidant activity of processed pomelo peels after oven drying (50 and $60{ }^{\circ} \mathrm{C}$ ) and freeze drying treatments in comparison with fresh pomelo peels. A slightly different $(4 \%-6 \%)$ in TPC was observed in oven dried pomelo peels in comparison with fresh peels, indicating that there was a slightly effect of post-drying methods on retaining the antioxidant potential of these natural byproducts. In vitro assays revealed that post-drying methods involved oven drying exerted a higher TPC compared to freeze drying in overall parts of pomelo byproducts. Highest retention of bioactive compounds was found in fresh pomelo peels (437-1876 mg GAE/100 g DM), followed by oven dried at 50 and $60{ }^{\circ} \mathrm{C}(892-$ $1336 \mathrm{mg} \mathrm{GAE} / 100 \mathrm{~g} \mathrm{DM}$ ), and freeze dried (555-1267 mg GAE/100 g DM). In terms of oven dried pomelo byproducts, the highest TPC was retained in albedo (1337 mg GAE/100 g DW), followed by flavedo (1226 mg GAE/100 g DW), lamella (998 mg GAE/100 g DW) and pulp waste (962 mg GAE/100 g DW) respectively. Therefore, oven drying was the most suitable post-drying method to retain the bioactive compounds for pomelo byproducts. This knowledge may inspire consumer or pomelo industry operators to re-evaluate their byproducts, reduce the waste and expand the application of pomelo fruits.
\end{abstract}

Keyword: Antioxidant; Drying; Pomelo peels; Total phenolic contents 